\title{
Immune cell infiltration and related core genes expression characteristics in eosinophilic and non-eosinophilic chronic rhinosinusitis with nasal polyps
}

\author{
GAOYUN XIONG ${ }^{1 *}$, XIAOXING XIE ${ }^{1 *}$, QINGLIANG WANG ${ }^{1}$, \\ YANYAN ZHANG ${ }^{1}$, YANPING GE ${ }^{1}$, WEI LIN ${ }^{1}$ and MINGQIAN $\mathrm{LI}^{2}$ \\ ${ }^{1}$ Department of Otolaryngology, Head and Neck Surgery; ${ }^{2}$ Cancer Institute of Integrated Traditional \\ Chinese and Western Medicine, Zhejiang Academy of Traditional Chinese Medicine, Tongde Hospital of \\ Zhejiang Province, Hangzhou, Zhejiang 310012, P.R. China
}

Received February 27, 2020; Accepted September 4, 2020

DOI: 10.3892/etm.2020.9310

\begin{abstract}
Chronic rhinosinusitis with nasal polyps (CRSwNP) refers to chronic inflammation of the sinonasal mucosa. It can either be eosinophilic (ECRSwNP) or non-eosinophilic (non-ECRSwNP). However, immune cell infiltration in the microenvironment and pathogenesis of ECRSwNP and non-ECRSwNP are still unclear. The aim of the present study was to assess the immune cell infiltration and molecular mechanisms of ECRSwNP and non-ECRSwNP. In the present study, 22 immune cell types in ECRSwNP and non-ECRSwNP were investigated by CIBERSORT based on transcriptome data. The core gene related pathophysiology of CRSwNP was analyzed using Weighted Gene Correlation Network Analysis according to the phenotype of the infiltrated eosinophils and nasal polyps (NP). A total of four types of immune cells (mast cells, activated dendritic cells, M2 macrophages and activated natural killer cells) were demonstrated to have a direct and indirect correlation with eosinophilic infiltration in ECRSwNP. M1 macrophages and activated $\mathrm{CD}^{+}{ }^{+}$memory $\mathrm{T}$ cells were
\end{abstract}

Correspondence to: Dr Mingqian Li, Cancer Institute of Integrated Traditional Chinese and Western Medicine, Zhejiang Academy of Traditional Chinese Medicine, Tongde Hospital of Zhejiang Province, 234 Gucui Road, Hangzhou, Zhejiang 310012, P.R. China E-mail: limingqian613@163.com

Dr Wei Lin, Department of Otolaryngology, Head and Neck Surgery, Zhejiang Academy of Traditional Chinese Medicine, Tongde Hospital of Zhejiang Province, 234 Gucui Road, Hangzhou, Zhejiang 310012, P.R. China

E-mail: lw205010@163.com

${ }^{*}$ Contributed equally

Key words: immune cell infiltration, eosinophilic chronic rhinosinusitis with nasal polyps, non-eosinophilic chronic rhinosinusitis with nasal polyps, gene expression, differential analysis correlated with major immune cell types in non-ECRSwNP. NP could affect the expression of 'olfactory receptor activity' and 'channel activity' genes to impair the olfactory signaling pathway and neuroactive ligand receptor pathway. 'Cell adhesion molecule binding', 'cytokine receptor binding' and 'glucocorticoid receptor binding' were significantly enriched in ECRSwNP, whereas epithelial cell injury, autophagy and the mTOR pathway (hsa04140 and hsa04150) may serve an important role in the pathogenesis of non-ECRSwNP. There were significantly different immune cell infiltration and related core genes expression characteristics between ECRSwNP and non-ECRSwNP. The results of the present study provide an improved basis for elucidation of the mechanism and treatment of CRSwNP.

\section{Introduction}

Chronic rhinosinusitis (CRS) is a multifactorial, heterogeneous inflammatory disease of the nose and paranasal sinuses characterized by 12 weeks of persistent symptoms, such as congestion, pain or facial pressure, stuffiness, cough, impairment or loss of the sense of smell (anosmia), nasal discharge and fatigue (1). CRS affects $\sim 12 \%$ of the worldwide population and severely impairs quality of life (2). Based on the absence or presence of nasal polyps (NP), CRS can be categorized into two main forms, CRS without NP(CRSsNP) and CRS with NP (CRSwNP) (1). CRSwNP is the most commonly studied among both types. Polyps are outgrowths of edematous inflammatory tissues that have grown into the middle meatus (3). CRSwNP causes anosmia more readily than CRSsNP and is less likely to respond to antibiotics $(1,4)$. CRSwNP can further be divided into two subtypes based on the histological features: Eosinophilic CRSwNP (ECRSwNP) and non-eosinophilic CRSwNP (non-ECRSwNP). In Western countries, $67-88 \%$ of patients with CRSwNP exhibit mucosal eosinophilic infiltration, which is characterized by type II inflammation (5-7). However, in East Asian countries, such as China, Korea and Japan, only $21.7-59.6 \%$ of patients exhibit mucosal eosinophilia, which is characterized by Thelper cell (Th)1/Th17-dominant inflammation (7-11). There was a significant increase in the number 
of patients with ECRSwNP compared with non-ECRSwNP from 1999 to 2011 in Thailand (12). ECRSwNP is higher in CT images and polyp scores compared with non-ECRSwNP and has a poor response to medical and surgical therapy $(9,13)$. The pathogenesis and pathophysiological mechanisms of ECRSwNP and non-ECRSwNP remain poorly understood.

Immune cell infiltration is the characteristic manifestation of chronic inflammation. A histological study on a large sample of CRS tissues demonstrated that CRSwNP presented a higher amount of immune cell infiltration compared with CRSsNP, and there were higher levels of cell infiltration of cytotoxic $\mathrm{CD}^{+} \mathrm{T}$ cells, B cells and macrophages in CRSwNP, as determined by immunohistochemistry (14). In addition, ECRSwNP has been reported to be associated with fewer M1 macrophages and more M2 macrophages compared with non-ECRSwNP, and the number of IL- $10^{+} \mathrm{CD} 68^{+}$macrophages was decreased in ECRSwNP (15). Specific chemokines and cytokines, such as MIP-1 $\alpha$, IL-5 and RANTES, are crucial to eosinophil recruitment, survival and differentiation (16-18). Based on gene expression profiles, Th2-related molecules (IL-4 and colony-stimulating factor 2), cell cycle regulators (cyclin dependent kinase inhibitor 1A and cyclin D1), and tissue fibrosis-related molecules (TGF- $\beta$ ) are upregulated in ECRSwNP; on the other hand, interferons (IFNs) and acute inflammatory cytokines are upregulated in non-ECRSwNP (19). The long non-coding RNA XLOC_010280 was demonstrated to be specifically expressed in ECRSwNP and found to regulate chemokine (C-C motif) ligand 18 (CCL18), which may explain the markedly higher expression of CCL18 in ECRSwNP compared with that in non-ECRSwNP (20).

To reveal the immune cell infiltration and key genes of CRSwNP, 22 immune cell types and the pathophysiology of CRSwNP were analyzed by CIBERSORT (21) and Weighted Gene Correlation Network Analysis (WGCNA) (22) methods according to eosinophilic pathology characteristics. The combined analysis of immune cell infiltration and key genes of CRSwNP provided a deeper understanding of the immune and inflammatory response exhibited in CRS.

\section{Materials and methods}

Acquisition and processing of transcriptome and gene expression profile data. The transcriptome gene expression profile data used in the present study were obtained from a public domain. The expression matrix and sample information (GSE72713) (20) were downloaded from the Gene Expression Omnibus (GEO) database (https://www.ncbi.nlm.nih. gov/geo/). Genes with similar names were merged and homogenized using the limma package (version 3.11; https://www. bioconductor.org/packages/release/bioc/html/limma.html). Genes that were not expressed in all samples were omitted; a total of 21,014 expressed genes were further analyzed in all samples.

Evaluation of immune cell infiltration in CRSwNP. The nine samples obtained from GSE72713 was used to investigate the 22 immune cell types in CRSwNP by CIBERSORT $(20,21)$. CIBERSORT is an analytical tool in which 547 gene expression signatures were used to quantify the 22 immune cells. The expression data were submitted to the CIBERSORT official website (https://cibersort.stanford.edu/). The algorithm used a signature matrix at 2,500 permutations to improve the accuracy of the deconvolution. Kruskal-Wallis test was used to analyze the differences among the three groups (Normal control, ECRSwNP and non-ECRSwNP) using R software (version 3.6.3; https://www.r-project.org/). The correlation of infiltrated immune cells was analyzed by a Pearson's test using corrplot R package (Version 0.84; https://github. com/taiyun/corrplot) (22).

Construction of the WGCNA phenotype co-expression network. The nine samples obtained from GSE72713 were divided into four groups: Normal control (CTRL; $n=3$; GSM1868859; GSM1868860; GSM1868861), ECRSwNP ( $\mathrm{n}=3$; GSM1868853; GSM1868854; GSM1868855), non-ECRSwNP $(\mathrm{n}=3$; GSM1868856; GSM1868857; GSM1868858), and NP, which included ECRSwNP and non-ECRSwNP samples. The tissue eosinophil number per high power field (HPF) of ECRSwNP was $>20$, counted in the lamina propria of the polyps in five random microscopic HPFs at $\mathrm{x} 400$ magnification. Subjects who did not fulfill the criteria were categorized as non-ECRSwNP. Overall, 21,014 genes from nine samples were analyzed by WGCNA (23). The samples were clustered and the pickSoftThreshold was calculated (24). Power value 16 was used to construct the block-wise modules (24). The color row beneath the dendrogram illustrates the 28-module assignment obtained using the dynamicTreeCut package (version 1.63) (25). The correlation between phenotype and block-wise modules was then analyzed using Pearson's test.

The gene expression levels of related modules were verified by quantitative PCR (qPCR). GAPDH was as the reference gene. The mRNA expression for the selected 12 genes was measured using SYBR ${ }^{\circledR}$ Premix Ex Taq ${ }^{\mathrm{TM}}$ (Takara Bio, Inc.) under the following thermocycling conditions: Initial denaturation at $95^{\circ} \mathrm{C}$ for $5 \mathrm{~min}$, followed by 40 cycles at $95^{\circ} \mathrm{C}$ for $10 \mathrm{sec}$ and $55^{\circ} \mathrm{C}$ for $30 \mathrm{sec}$. The expression levels in the samples were determined by the relative quantity curve method $2^{-\Delta \Delta \mathrm{Cq}}(26)$. Primer sequences are listed in Table SI. The new collected qPCR verified samples (non-eosinophil polyps, eosinophil and nasal mucosa tissues), which are distinct from the nine samples aforementioned, were collected by the Department of Otolaryngology, Head and Neck Surgery, Tongde Hospital of Zhejiang Province (Zhejiang, China) between April 2018 and December 2019. The Ethics Committee of Tongde Hospital of Zhejiang Province (approval no. XMSB2018013) approved the present study and written informed consent was obtained from nine patients (mean age: $34 \pm 5$ years; age range: $25-40$ years; nine males and three females). The RNA was extracted from the nine tissue samples of patients using RNeasy Mini Kit (Qiagen $\mathrm{GmbH}$ ) according to manufacturer's protocol and cDNA was synthesized using First Strand cDNA Synthesis Kit (cat. no. K1612; Thermo Fisher Scientific, Inc.). The 12 related genes were amplified by qPCR using Roche LightCycler ${ }^{\circledR}$ 480II (Roche diagnostics).

Module gene function analysis. The gene functions of modules that were found to be significantly related to the phenotypes (CTRL, ECRSwNP, non-ECRSwNP and NP) were enriched in Gene Ontology (GO; http://geneontology.org/) and Kyoto Encyclopedia of Genes and Genomes (KEGG) 


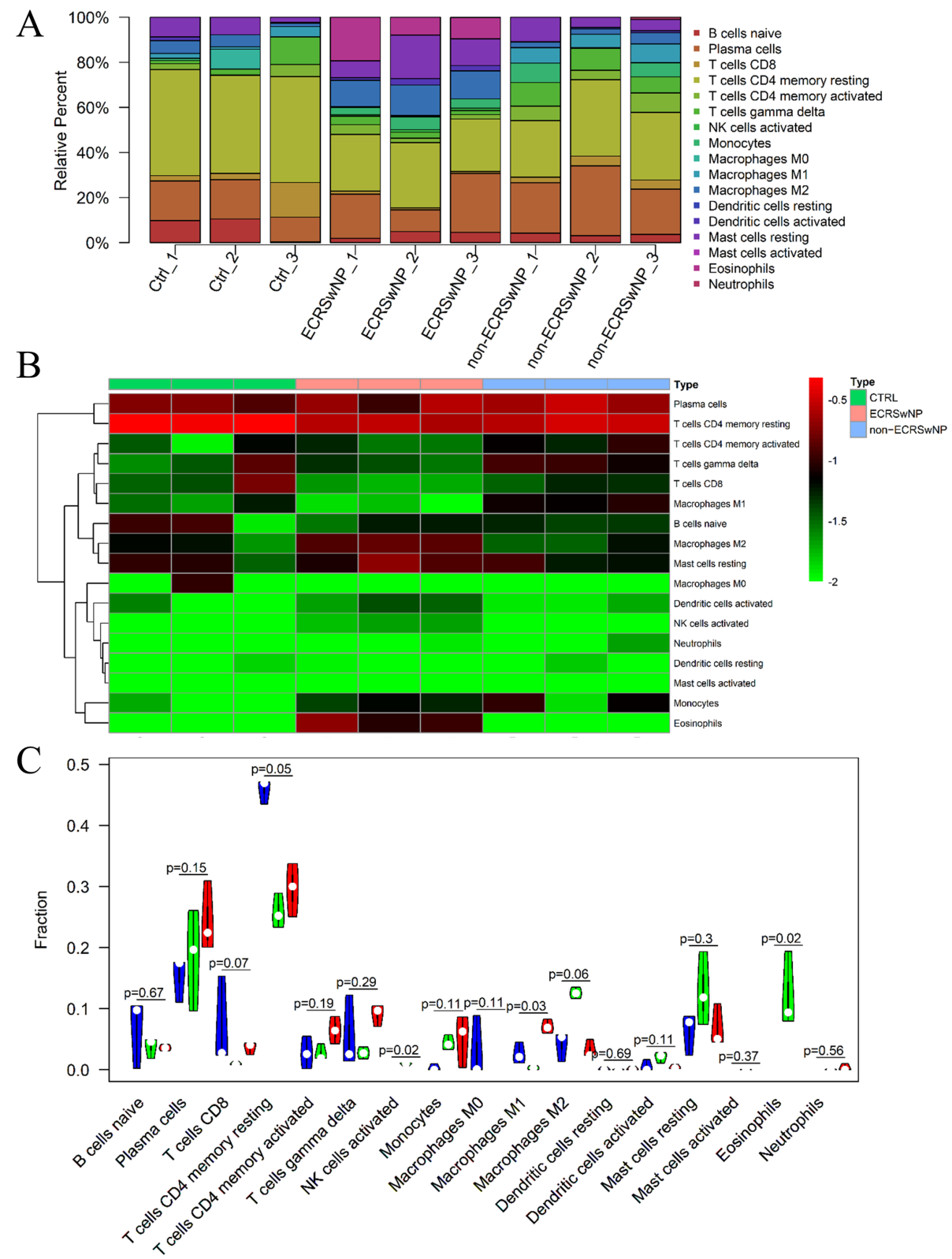

Figure 1. Composition of infiltrated immune cells among CTRL, ECRSwNP and non-ECRSwNP specimens as obtained via CIBERSORT used at a signature matrix of 2,500 permutations. (A) Bar plot of proportions of 22 immune cell infiltrated in CTRL, ECRSwNP and non-ECRSwNP groups. (B) Heatmap and cluster of 22 immune cell types infiltrated in CTRL, ECRSwNP and non-ECRSwNP. (C) Violin plot of the proportions of 17 immune cells infiltrated in the CTRL (blue), ECRSwNP (green) and non-ECRSwNP (red) groups. The horizontal axis shows the clustering information of the samples. ECRSwNP, eosinophilic chronic rhinosinusitis with nasal polyps; non-ECRSwNP, non-eosinophilic chronic rhinosinusitis with nasal polyps; CTRL, control.

(https://www.kegg.jp/). P $<0.05$ was set as the cut-off value. Connections between the significant GO and KEGG terms were visualized using a clusterProfiler package with a network diagram (27). The heatmap was drawn using the pheatmap package (version 1.0.12; https://cran.r-project.org/web/packages/pheatmap/index.html).

\section{Results}

Composition of immune cells in CRSwNP. Initially, the fractions of infiltrated immune cells among the CTRL, ECRSwNP and non-ECRSwNP groups were investigated based on the gene expression profile data by CIBERSORT. The percentage of infiltrated cells was shown and 17 types of immune cells were found to infiltrate the nasal mucosa and NP tissues
(Table SII). A total of five types of immune cells (memory B cells, naive CD4+ T cells, follicular helper T cells, regulatory $\mathrm{T}$ cells and resting NK cells) were not found in the CTRL, ECRSwNP and non-ECRSwNP groups (Fig. 1 and Table SII). A notable difference was observed in eosinophil infiltration among the CTRL, ECRSwNP and non-ECRSwNP groups (Fig. 1). In addition, the heatmap depicted plasma and $\mathrm{CD}^{+}$ memory resting cells as the major cell groups using the pheatmap package (Fig. 1B). The percentage of eosinophils in the infiltrated cells was $12.26 \pm 5.08 \%$ in the ECRSwNP group, but no eosinophils infiltrated the CTRL and non-ECRSwNP groups (Fig. 1 and Table SII). The activated natural killer (NK) cells were also only present in the ECRSwNP group (Fig. 1B). Although the number of infiltrated resting $\mathrm{CD}^{+} \mathrm{T}$ cells in CTRL tissues $(45.86 \pm 1.67 \%)$ was higher compared 




Figure 2. Correlation matrix of the proportion of 22 immune cells in the CTRL, ECRSwNP and non-ECRSwNP groups. Variables are ordered in the heatmap matrix. Eosinophils were significantly positively correlated with activated mast cells, activated NK cells and M2 macrophages. The M2 macrophages were significantly positively correlated with eosinophils, activated NK cells and activated dendritic cells, and significantly negatively correlated with $\gamma \delta \mathrm{T}$ cells and M1 macrophages. The M1 macrophages were significantly positively correlated with $\gamma \delta \mathrm{T}$ cells and activated CD4 ${ }^{+} \mathrm{T}$ cells. In the heatmap, the blue color represents low adjacency (negative correlation), while the red represents high adjacency (positive correlation). ECRSwNP, eosinophilic chronic rhinosinusitis with nasal polyps; non-ECRSwNP, non-eosinophilic chronic rhinosinusitis with nasal polyps; CTRL, control; NK, natural killer.

with that in ECRSwNP $(25.83 \pm 2.32 \%)$ and non-ECRSwNP $(29.62 \pm 3.56 \%)$, the infiltrated percentage of activated $\mathrm{CD} 4^{+}$ $\mathrm{T}$ cells in the non-ECRSwNP group $(6.46 \pm 1.84 \%)$ was higher compared with that in the ECRSwNP $(2.68 \pm 1.11 \%)$ and CTRL $(2.74 \pm 2.18 \%)$ groups (Fig. 1C and Table SII). Among infiltrated macrophages (M0, M1 and M2), the number of infiltrated M2 macrophages in the ECRSwNP group $(12.56 \pm 0.77 \%)$ was higher compared with that in the CTRL $(4.12 \pm 1.98 \%)$ and non-ECRSwNP $(3.24 \pm 1.21 \%)$ groups. Additionally, the number of infiltrated M1 macrophages in the non-ECRSwNP group $(7.06 \pm 0.9 \%)$ was higher compared with that in the CTRL $(2.58 \pm 1.43 \%)$ and ECRSwNP $(0.31 \pm 0.26 \%)$ groups. M0 macrophage infiltration was found in lesser amounts in the CTRL group, but not in the other two CRSwNP groups. Furthermore, the activated dendritic cells $(2.06 \pm 0.72 \%)$ and resting mast cells $(12.86 \pm 5.92 \%)$ in the ECRSwNP group were higher compared with those in the CTRL (activated dendritic cells $0.56 \pm 0.78 \%$; resting mast cells $6.29 \pm 2.81 \%$ ) and non-ECRSwNP groups (activated dendritic cells $6.79 \pm 2.86 \%$; resting mast cells $0.43 \pm 0.31 \%$; Fig. 1 and Table SII).
Correlation among infiltrated immune cells in CRSwNP. A total of five immune cell types, including eosinophils, activated NK cells, activated dendritic cells, resting mast cells and M2 macrophages, were found in higher fractions in the ECRSwNP group compared with those in the CTRL and non-ECRSwNP groups (Fig. 1C and Table SII). The correlation between these infiltrated cells was further analyzed. Eosinophils were found to be significantly positively correlated with activated mast cells, activated NK cells and M2 macrophages ( $r>0.75$; Fig. 2). The M2 macrophages were significantly positively correlated with eosinophils, activated NK cells and activated dendritic cells ( $r>0.75$ ), but significantly negatively correlated with $\gamma \delta \mathrm{T}$ cells and M1 macrophages $(\mathrm{r}<-0.75$; Fig. 2). The M1 macrophages were significantly positively correlated with $\gamma \delta \mathrm{T}$ cells and activated $\mathrm{CD} 4^{+} \mathrm{T}$ cells ( $r>0.75$; Fig. 2). These three cell types mostly infiltrated the non-ECRSwNP group (Fig. 1 and Table SII). Thus, these results suggested that ECRSwNP and non-ECRSwNP may differ significantly in immune cell infiltration and core gene expression profiles. 

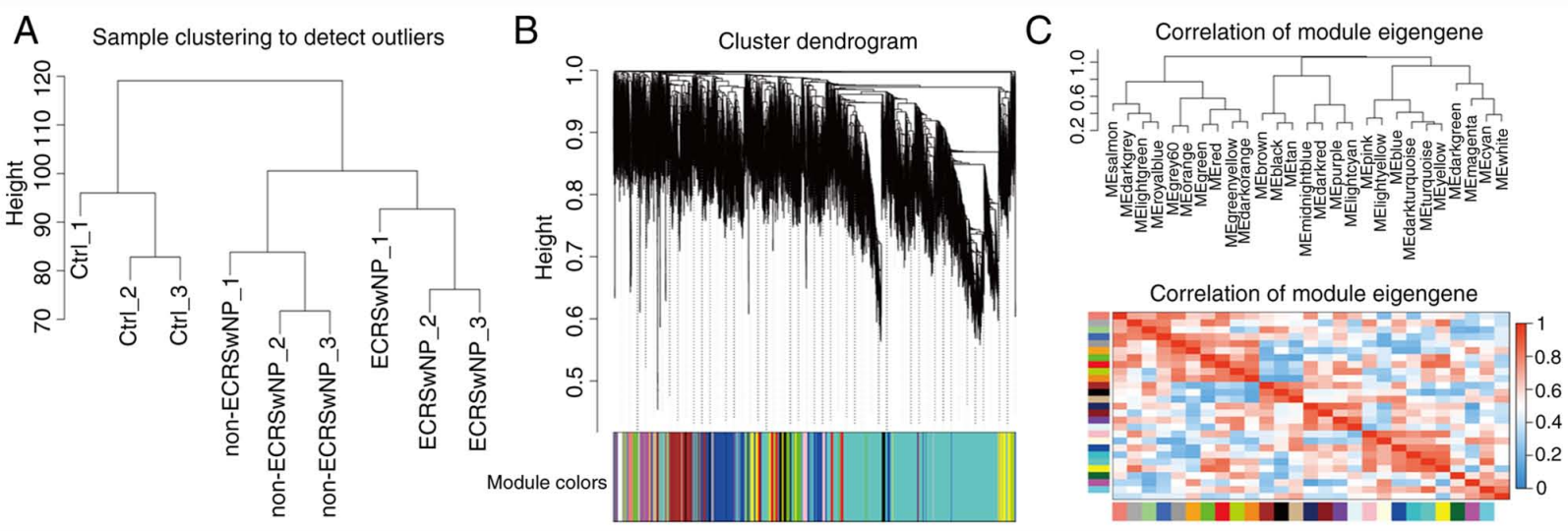

Figure 3. Gene expression module analysis in the CTRL, ECRSwNP and non-ECRSwNP groups. (A) CTRL, ECRSwNP and non-ECRSwNP groups were clustered by hierarchical cluster analysis. The three groups of samples showed good expression clustering. (B) Gene dendrogram obtained by average linkage hierarchical clustering in the CTRL, ECRSwNP and non-ECRSwNP groups. The color row beneath the dendrogram illustrates the 28-module assignment obtained by the dynamicTreeCut package. (C) Hierarchical clustering and correlation of 27 gene modules that summarizes the modules found in clustering analysis. Branches of the dendrogram (the meta-modules) group eigengenes that are positively correlated. ECRSwNP, eosinophilic chronic rhinosinusitis with nasal polyps; non-ECRSwNP, non-eosinophilic chronic rhinosinusitis with nasal polyps; CTRL, control.

Core gene expression modules in CRSwNP. To detect different core genes in ECRSwNP and non-ECRSwNP, gene expression profiles of the three group samples (CTRL, ECRSwNP and non-ECRSwNP) involving 21,014 genes were clustered by hierarchical cluster analysis. Good expression clustering was observed in all three groups (Fig. 3A). In addition, power value 16 was used to construct block-wise modules using WGCNA. The 21,014 expressed genes were divided into 28 modules according to expression (Fig. 3B and C). The grey module was the unknown module. The expression levels of each module gene were clustered and correlated. All gene modules were clustered into three big clades (Fig. 3B and C). The gene expression modules and clades may be closely related to the phenotype of CRSwNP.

Correlation of gene expression modules with the phenotype of $C R S w N P$. To clarify the correlation between the gene module and phenotype, the obtained samples were divided into four groups (CTRL, ECRSwNP, non-ECRSwNP and NP groups). Two gene modules (pink and turquoise) were found to be significantly negatively correlated with the nasal polyp phenotype $(\mathrm{P}<0.05 ; \mathrm{r}<-0.80 ;$ Fig. 4). The gene function clusters of the two modules were analyzed by clusterProfiler for GO and KEGG. The pink module showed no significant enrichment of GO functions and KEGG pathways. The turquoise module was significantly enriched $(\mathrm{P}<0.05)$ in $64 \mathrm{GO}$ terms and seven KEGG pathways (Fig. 5 and Table SIII). The 'olfactory receptor activity' (GO:0004984) and 'olfactory transduction' (hsa04740) were major functional enrichments (Fig. 5A and $\mathrm{C}$ ). The $\mathrm{G}$ protein-coupled receptor activity and channel proteins activity in turquoise module have extensive networks of shared genes (Fig. 5B). The expression of the olfactory related genes, including OR10A3 and OR1A1, was higher in CTRL groups compared with that in the NP group, especially in ECRSwNP (Figs. 5D and S1). These results revealed the molecular mechanism by which CRSwNP affects olfaction.

Correlation of gene expression modules with ECRSwNP and non-ECRSwNP. The gene module and phenotype correlation analysis showed that the brown module was significantly positively correlated with ECRSwNP (P=0.003; $\mathrm{r}=0.86$; Fig. 4). In the brown module, $20 \mathrm{GO}$ terms and $16 \mathrm{KEGG}$ pathways were significantly enriched $(\mathrm{P}<0.05$; Fig. 6 and Table SIV). The 'CCR chemokine receptor binding' (GO:0048020), 'cytokine activity' (GO:0005125), 'cytokine receptor binding' (GO:0005126), 'receptor ligand activity' (GO:0048018), 'growth factor receptor binding' (GO:0070851) and 'epidermal growth factor receptor binding' (GO:0005154) function (Fig. 6A) formed a functional network that was significantly positively associated (Figs. 4 and 6B). In the brown module, many genes, including $\mathrm{CDH} 5, \mathrm{SHC} 1$ and ITGA5, were found to be associated with 'cytokine receptor binding' and 'growth factor receptor binding' double GO function (Fig. 6C). Multiple inflammatory-related signaling pathways, including, PI3K-Akt signaling, JAK-STAT signaling, TNF and IL-17 signaling, were significantly enriched in the brown module (Fig. 6D). The gene expression of the brown module in the ECRSwNP group was higher compared with that in the CTRL and non-ECRSwNP groups (Fig. 6E and F). The GO function and enriched pathways illustrated the molecular mechanism of ECRSwNP formation.

The correlation analysis did not demonstrate the significant positive correlation of a gene module with non-ECRSwNP, although it justified the significant negative correlation of the blue module with non-ECRSwNP $(\mathrm{P}<0.05 ; \mathrm{r}<-0.80$; Fig. 4). The GO analysis revealed that genes correlated with non-ECRSwNP mainly involved a variety of transferases (GO:0034212, GO:0004674, GO:0018024, GO:0019787 and GO:0004842), GTPases (GO:0031267, GO:0030695, GO:0017016 and GO:0005096) and receptor binding functions (GO:0046965, GO:0046966, and GO:0035257; Fig. 7A and Table SV). These functional genes formed four major functional networks (Fig. 7B). Numerous cell proliferation-related and cancer signaling pathways, such as the 'MAPK signaling pathway', 'Wnt signaling pathway', 'mTOR signaling pathway', 'hepatocellular carcinoma' and 'breast cancer' in the blue module and some suppressor genes (such as P53 and DUSP8) involved in these pathways were downregulated 


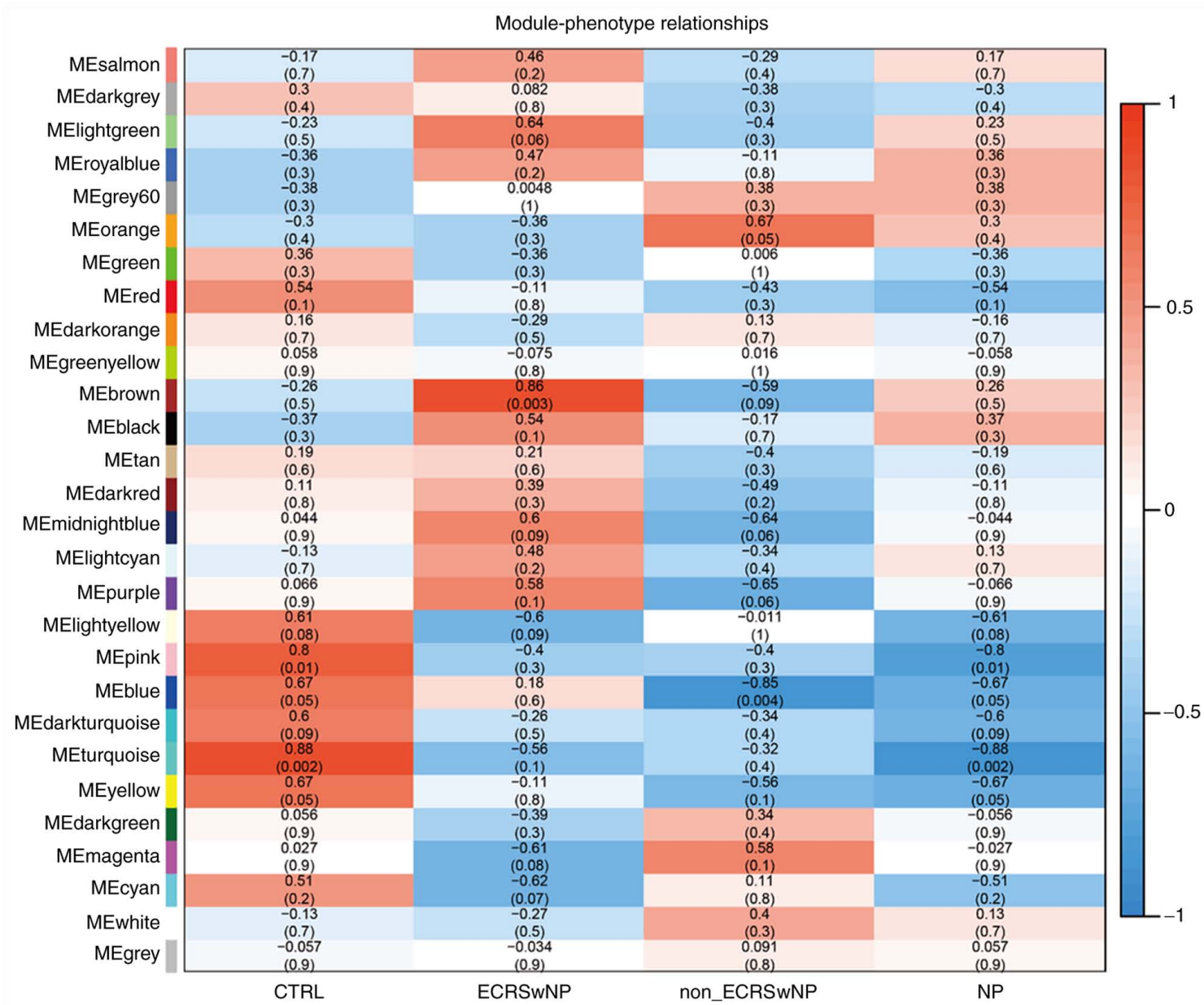

Figure 4. Heatmap plot showing correlation of the gene module with phenotypes of CRSwNP. Each row in the heatmap corresponds to one gene module (labeled by color). The column corresponds to one phenotype. In the heatmap, the blue color represents low adjacency (negative correlation), while the red represents high adjacency (positive correlation). ECRSwNP, eosinophilic chronic rhinosinusitis with nasal polyps; CTRL, control; NP, nasal polyps; ME, module eigengene.

in non-ECRSwNP group compared with those in the CTRL and ECRSwNP groups (Fig. 7C and E). There were extensive networks of interactions between these gene signaling pathways (Fig. 7D). These results suggested that the formation of non-ECRSwNP was similar to the growth of tumors (Fig. 7E). However, the 'autophagy' (hsa04136, hsa04140 and hsa04150) was found to be negatively associated with non-ECRSwNP (Figs. 4 and 7C), where some autophagy-related genes (such as CAMKK2, ATG2A and MAPK9) were downregulated in non-ECRSwNP compared with those in the CTRL and ECRSwNP groups (Fig. 7F). The dysfunction of autophagy in nasal mucosa may be the primary cause of non-ECRSwNP.

\section{Discussion}

CRS is defined as inflammation of the nasal mucosa; CRSwNP represents $20-25 \%$ of all cases worldwide (3). The two main histological types of CRSwNP (ECRSwNP and non-ECRSwNP) have been identified based on eosinophil infiltration, geographical location and ethnicity (5-11). The immune cell infiltration of CRSwNP is higher compared with that of CRSsNP (14); however, the rate of infiltration of different types of immune cells, other than eosinophils and macrophages, is still unclear in ECRSwNP and non-ECRSwNP (15). In the present study, the proportion of 22 immune cell types in immune microenvironment of ECRSwNP and non-ECRSwNP was analyzed by CIBERSORT based on the gene expression profile.

The resting $\mathrm{CD}^{+}$memory $\mathrm{T}$ cells were found in the highest proportion in the CTRL group, whereas the activated $\mathrm{CD}^{+} \mathrm{T}$ cells were mainly infiltrated in the non-ECRSwNP group. Memory $\mathrm{T}$ cells are essential components of immunological memory, and memory CD4 cells could help protect against infections (28). The memory $\mathrm{CD}^{+} \mathrm{T}$ cells also outnumber memory $\mathrm{CD}^{+} \mathrm{T}$ cells in the lung, skin and mucosal surfaces, and function to direct protective responses in addition to coordinating the recruitment of immune cells to tissue sites (29-31). The resident $\mathrm{CD}^{+}$memory $\mathrm{T}$ cells remodel epithelial responses to accelerate neutrophil recruitment during pneumonia (32). These findings indicated that the occurrence of non-ECRSwNP may be closely related to the activation of $\mathrm{CD}^{+}$cells. The activated $\mathrm{CD}^{+} \mathrm{T}$ cells were positively correlated with M1 macrophages and $\gamma \delta \mathrm{T}$ cells. Non-ECRSwNP has been reported to be characterized by Th1/Th17-dominant inflammation, and infiltration by a greater number of neutrophils and M1 macrophages $(1,14,15)$. IFN- $\gamma$, which promotes the polarization of M1 macrophages, is highly expressed in non-ECRSwNP (33). The functionality of $\gamma \delta \mathrm{T}$ cells is induced upon the recognition of stress antigens, 



Figure 5. Functional annotation and expression of the turquoise gene module of the CTRL group in Fig. 4 that is significantly negatively correlated with the nasal polyp phenotype, using the threshold of $\mathrm{P}<0.05$ and $\mathrm{r}>0.80$. (A) Significantly enriched GO terms in the turquoise gene module. (B) The network of gene overlap enriched GO terms in the turquoise gene module. (C) Significantly enriched Kyoto Encyclopedia of Genes and Genomes pathways in the turquoise gene module. The vertical items include the GO term name, whilst the length of the horizontal graph represents the gene ratio. The depth of the color represents the adjusted P-value in A-C. (D) Gene expression heatmap of olfactory receptor activity and olfactory transduction pathway. GO, Gene Ontology; CTRL, control.

which promotes cytokine production, and regulates pathogen clearance, inflammation and tissue homeostasis (34).

In the ECRSwNP group, the eosinophil infiltration was significantly correlated with the infiltration of M2 macrophages, activated mast cells and activated NK cells. Enhanced accumulation of M2 macrophages has previously been demonstrated in ECRSwNP compared with in non-ECRSwNP $(15,35)$. The crucial role of mast cells was evident in a mouse model of eosinophilic CRS, where none of the mast cell-deficient mice subjected to chronic allergen challenge developed cystic changes or polypoid changes in the nose or sinuses $(36,37)$. Furthermore, in a previous study, patients with CRS presented a significant decrease in eosinophil apoptosis mediated by NK cells compared with healthy controls; eosinophilic inflammation and NK cell dysfunction were responsible for this decrease (38).

Gene expression was correlated with phenotype to obtain gene expression modules for NP, ECRSwNP and non-ECRSwNP groups in the present study. One previous study revealed that olfactory dysfunction is very frequent $(\sim 90 \%)$ in CRSwNP and does not depend on nasal obstruction, as assessed by both polyp size and nasal airflow limitation (39). A previous study established that superior turbinate eosinophilia is correlated with an olfactory deficit in patients with CRS (40). The enriched results of olfactory and channel activity genes suggested that NP may affect the expression of olfactory receptors and channel activity genes to impair the olfactory signaling and neuroactive ligand-receptor pathways. To the best of our knowledge, this is the first time that NP have been linked to the expression of olfactory genes.

The present study showed that in ECRSwNP, functions including the 'binding of cell adhesion molecules' (GO:0050839), 'DNA-binding transcription activator activity' (GO:0001228), 'cytokine receptor binding' (GO:0005126), 'cytokine activity' (GO:0005125), 'growth factor receptor binding' (GO:0005154) and 'glucocorticoid receptor binding' (GO:0035259), and 'inflammatory signaling pathways' 

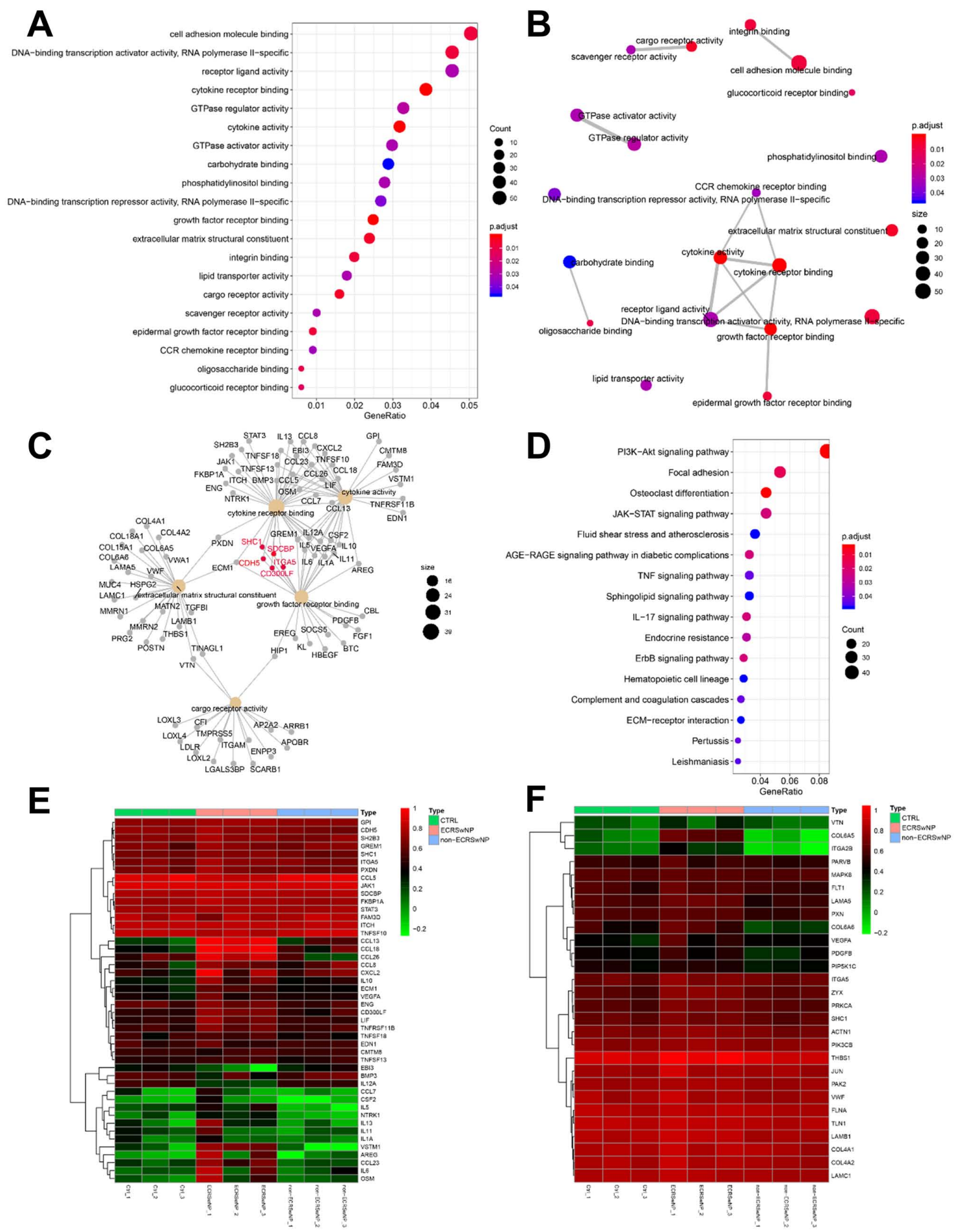

Figure 6. Functional annotation and expression of the brown gene module that is significantly positively correlated with eosinophilic chronic rhinosinusitis with nasal polyps. (A) Significantly enriched GO terms in the brown gene module. (B) The network of gene overlap enriched GO terms in brown gene module. (C) Gene correlation between most prominent GO term. (D) Significantly enriched Kyoto Encyclopedia of Genes and Genomes pathways in the brown gene module. The vertical items represent the GO term name, whereas the length of the horizontal graph represents the gene ratio. The depth of the color represents the adjusted P-value in A-D. (E) The gene expression heatmap of cytokine activity and cytokine receptor binding. (F) The gene expression heatmap of the focal adhesion pathway. GO, Gene Ontology.

(hsa04151, hsa04630 and hsa04668) were enriched and expressed a positive correlation. Cytokines are involved in the pathogenesis of CRS (41). IL-5 serves an important role in regulating eosinophil development, and is essential for its maturation and release into circulation (42-44). IL-13 can increase the levels of $\beta$-catenin, which contributes to cell-cell adhesion in CRS (45). The C-C chemokines, RANTES/CCL5, monocyte chemotactic protein-4 (MCP-4/CCL13), and eotaxin/CCL26 have also been found to be increased in atopic dermatitis skin lesions, and likely contribute to the chemotaxis of eosinophils 

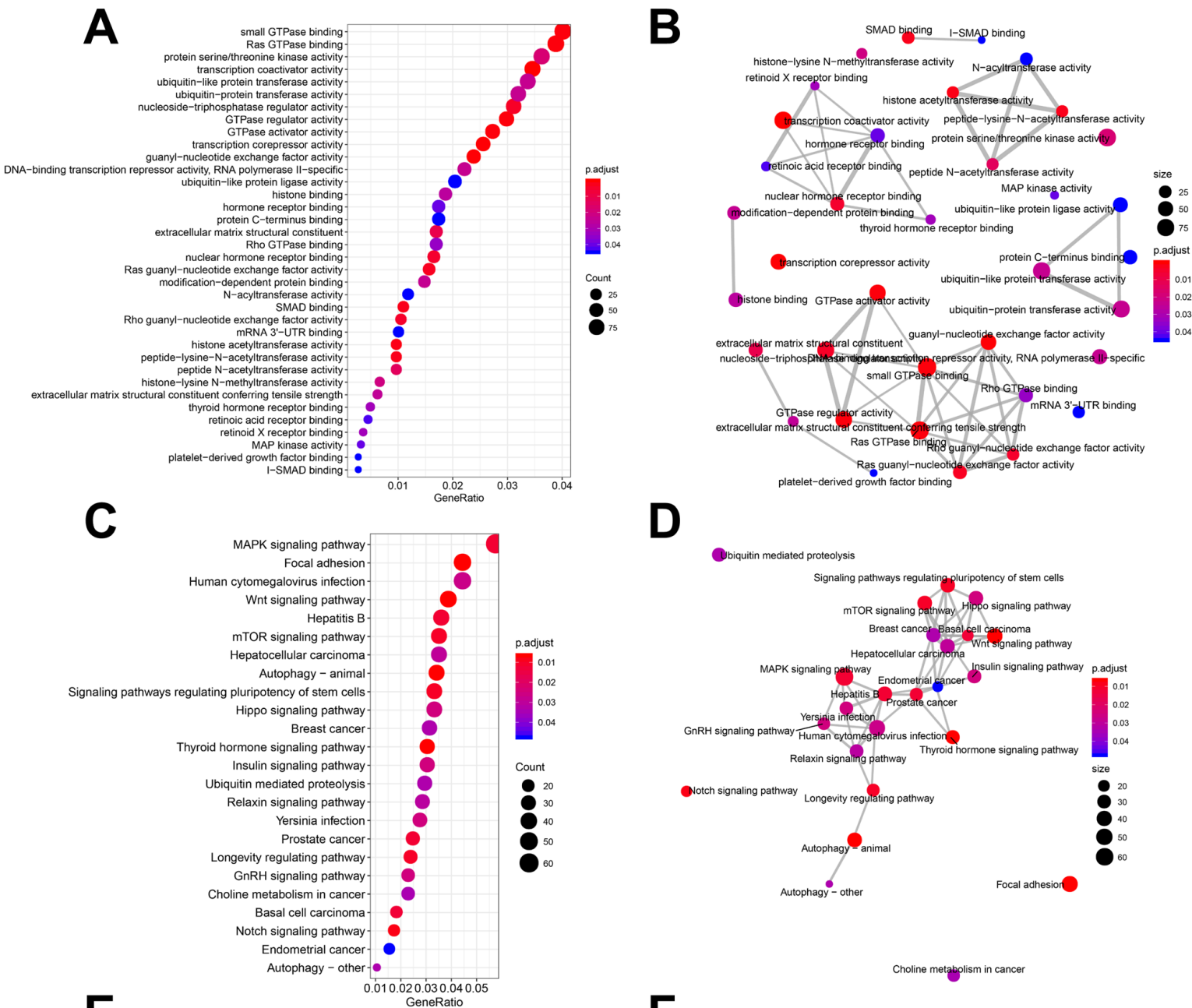

D



E
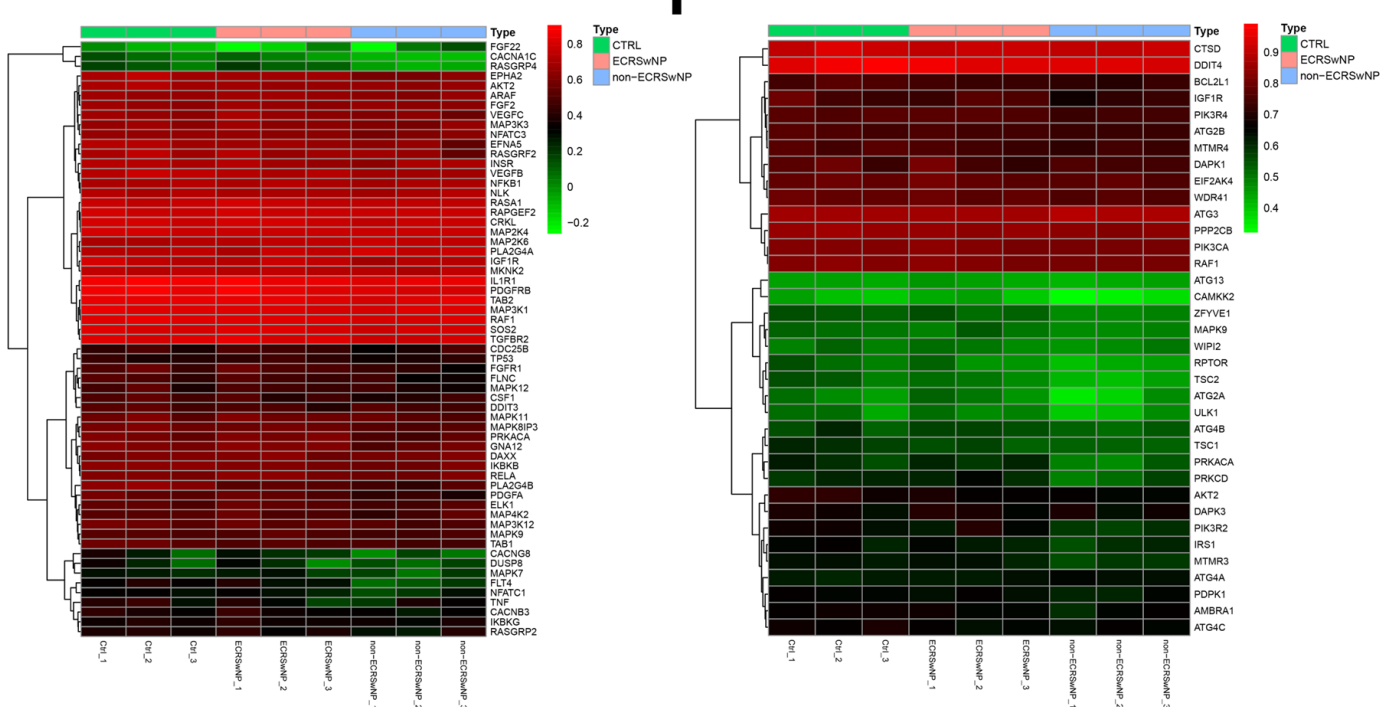

Figure 7. Functional annotation and expression of the blue gene module of the non-ECRSwNP group in Fig. 4 that is significantly negatively correlated with the eosinophilic chronic rhinosinusitis with nasal polyps, using the threshold of $\mathrm{P}<0.05$ and $\mathrm{r}<-0.80$. (A) Significantly enriched GO terms in blue gene module. (B) The network of gene overlap enriched GO terms in the blue gene module. (C) Significantly enriched KEGG pathways in the blue gene module. (D) The network of gene overlapping enriched KEGG terms in the blue gene module. The vertical items represent the GO term name, whereas the length of the horizontal graph represents the gene ratio. The depth of the color represents the adjusted P-value in (A-D) (E) The gene expression heatmap of the MAPK signaling pathway. (F) The gene expression heatmap of the autophagy-animal pathway. GO, Gene Ontology; KEGG, Kyoto Encyclopedia of Genes and Genomes; ECRSwNP, eosinophilic chronic rhinosinusitis with nasal polyps.

and Th2 lymphocytes into the skin (46). Oncostatin M, a member of the IL- 6 family, and IL- 6 , have been reported to serve an important role in the pathophysiology of eosinophilic inflammation in ECRSwNP (20). Nevertheless, controversy remains concerning IL- 6 function because IL- 6 has been predicted as an upstream regulator in non-ECRSwNP (19). 


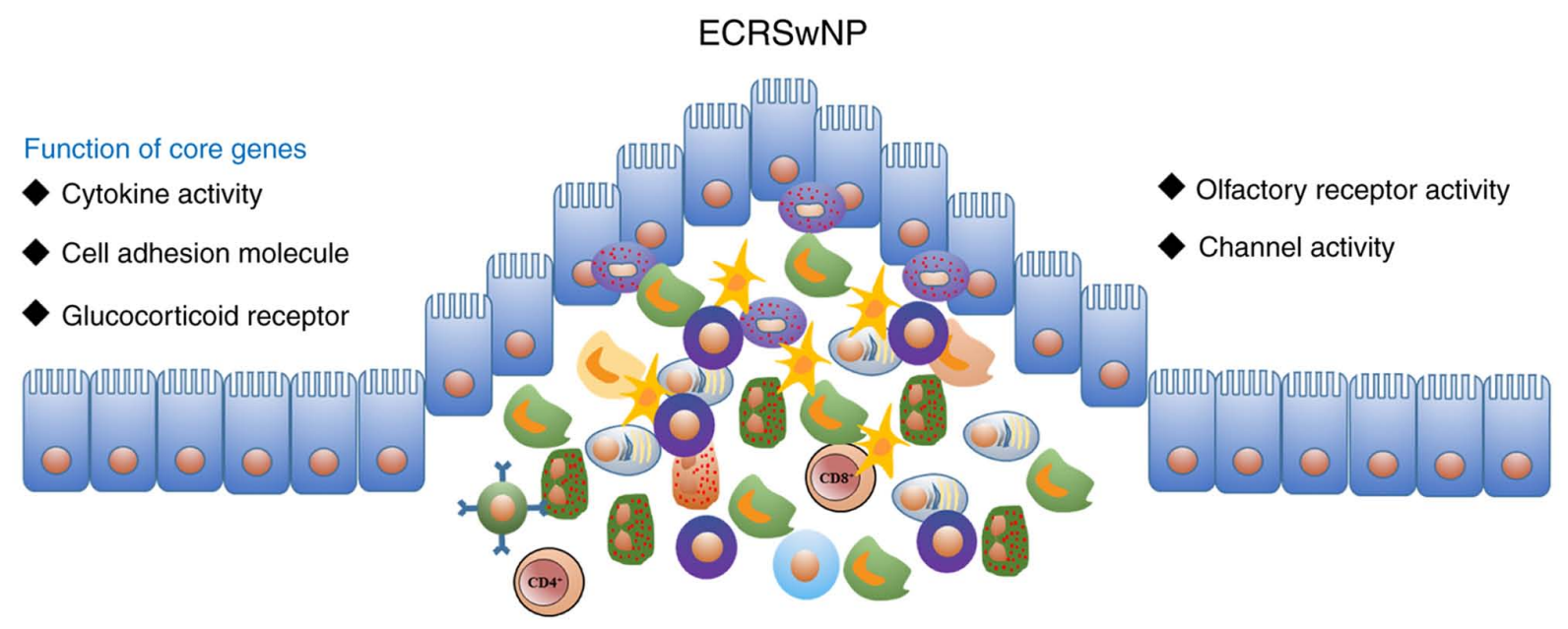

non-ECRSwNP

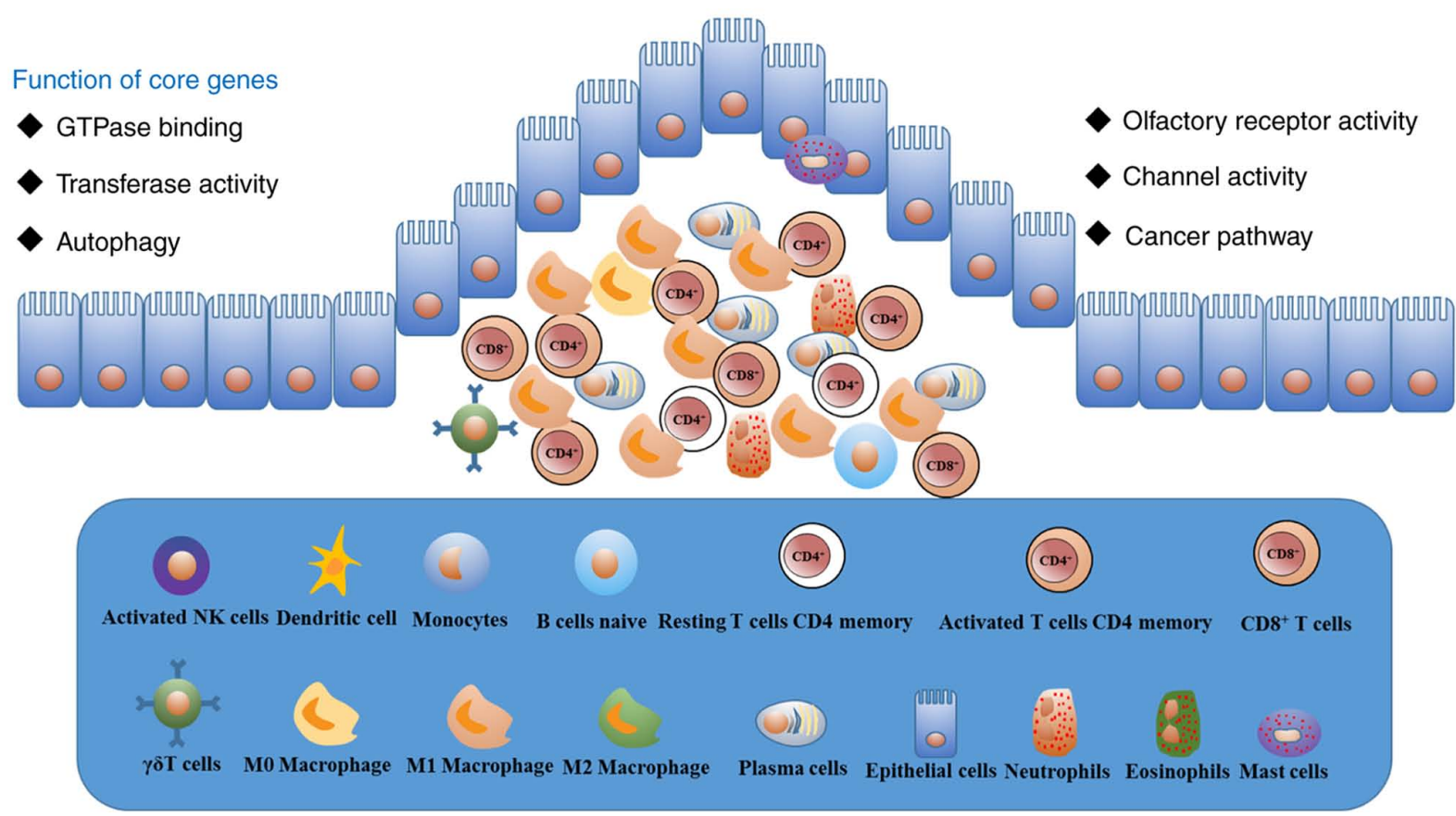

Figure 8. Significantly different immune cell infiltration and core genes of phenotypes between ECRSwNP and non-ECRSwNP were observed in the study. Four types of immune cells (activated mast cells, activated dendritic cells, M2 macrophages and activated NK cells) were directly and indirectly correlated with eosinophil infiltration in ECRSwNP. M1 macrophages and activated CD4 ${ }^{+}$memory $\mathrm{T}$ cells were correlated with major immune cell types in non-ECRSwNP. NP could affect the expression of olfactory receptor and channel activity genes to impair the olfactory signaling pathway and neuroactive ligand-receptor pathway. The cell adhesion molecule, cytokine and glucocorticoid receptors were significantly enriched in ECRSwNP, while the epithelial cell injury and autophagy may serve an important role in the pathogenesis of non-ECRSwNP. ECRSwNP, eosinophilic chronic rhinosinusitis with nasal polyps; non-ECRSwNP, non-eosinophilic chronic rhinosinusitis with nasal polyps; NK, natural killer.

The aforementioned cytokines were expressed in higher amounts in ECRSwNP compared with those in the CTRL and non-ECRSwNP groups. Topical corticosteroids have been demonstrated to exert a beneficial effect on ECRS and nasal polyposis, and the mechanism of action of nasal steroids appears to be multi-factorial, being initiated by their binding to the glucocorticoid receptor $(47,48)$. Glucocorticoid resistance to glucocorticoids may be the major cause of therapy failure in ECRSwNP (49).

Previous studies have demonstrated that in non-ECRSwNP, serum amyloid A (SAA) levels were significantly upregulated compared with those in ECRSwNP $(19,20)$; SAA triggers the production of cytokines associated with neutrophilic inflammation and improves neutrophil chemotaxis $(50,51)$. In the present study, the blue gene module was negatively correlated with non-ECRSwNP and established that genes correlated with non-ECRSwNP were mainly associated with a variety of transferases (GO:0034212, GO:0004674, GO:0018024, GO:0019787 and GO:0004842), GTPases (GO:0031267, GO:0030695, GO:0017016 and GO:0005096) and receptor binding functions (GO:0046965, GO:0046966, and GO:0035257) and cancer cell pathways (hsa05224, hsa05213, hsa05217 and hsa05215). These results suggested that the formation of non-ECRSwNP may be closely related to epithelial cell injury. Besides, it was identified 
that the autophagy pathway was negatively associated with non-ECRSwNP, and some autophagy genes were downregulated. It has recently been established that deficient autophagy in myeloid cells exacerbates CRS and that IFN- $\gamma$-induced insufficient autophagy adds to p62-dependent apoptosis of epithelial cells in CRSwNP $(52,53)$. Thus, these results suggested the significance of autophagy in non-ECRSwNP.

The composition and characteristics of infiltrated immune cells in the immune microenvironment of ECRSwNP and non-ECRSwNP were systematically analyzed in the present study (Fig. 8). A total of four types of immune cells (resting mast cells, activated dendritic cells, M2 macrophages and activated NK cells) were found to have a direct and indirect correlation with eosinophils in ECRSwNP. Notably, M1 macrophages and activated $\mathrm{CD}^{+}$memory $\mathrm{T}$ cells were correlated in non-ECRSwNP. The findings suggested that NP may affect the expression of olfactory receptors and channel activity genes to impair olfactory signaling pathways and neuroactive ligand-receptor pathways. In addition, the cell adhesion molecules, cytokines and glucocorticoid receptors may serve a vital role in ECRSwNP. On the other hand, epithelial cell injury and autophagy may serve an essential role in non-ECRSwNP. These results provided a good basis for the elucidation of the underlying mechanism and treatment of CRSwNP. However, the findings of this study were limited, as only a few high-quality sequencing samples were available in the public database. Thus, further analysis involving a large clinical sample is required.

\section{Acknowledgements}

Not applicable.

\section{Funding}

The present study was supported by grants from the National Natural Science Foundation of China (grant no. 81673809), the Scientific and Technological Program of Zhejiang province (grant no. 2017F10024), the Traditional Chinese Medicine Scientific Program of Zhejiang province (grant no. 2016ZQ002), the National Natural Science Foundation of Zhejiang province (grant no. LY19H280005) and the Medical and health Scientific and Technological Program of Zhejiang province (grant no. 2019KY347).

\section{Availability of data and materials}

The datasets used and/or analyzed during the present study are available from the corresponding author on reasonable request.

\section{Authors' contributions}

GX, XX, QW, YZ, YG, WL and ML designed the study and analyzed the data. ML, GX and XX wrote the manuscript. All authors read and approved the final manuscript.

\section{Ethics approval and consent to participate}

This study was approved by the Ethics Committee of Tongde Hospital of Zhejiang Province (approval no. XMSB2018013) and written informed consent was obtained from all patients.

\section{Patient consent for publication}

Not applicable.

\section{Competing interests}

The authors declare that they have no competing interests.

\section{References}

1. Fokkens WJ, Lund VJ, Mullol J, Bachert C, Alobid I, Baroody F, Cohen N, Cervin A, Douglas R, Gevaert P, et al: European position paper on rhinosinusitis and nasal polyps 2012. Rhinol Suppl 23: 1-298, 2012.

2. Kwah JH and Peters AT: Nasal polyps and rhinosinusitis. Allergy Asthma Proc 40: 380-384, 2019.

3. Schleimer RP: Immunopathogenesis of chronic rhinosinusitis and nasal polyposis. Annu Rev Pathol 12: 331-357, 2017.

4. Meltzer EO, Hamilos DL, Hadley JA, Lanza DC, Marple BF, Nicklas RA, Bachert C, Baraniuk J, Baroody FM, Benninger MS, et al: Rhinosinusitis: Establishing definitions for clinical research and patient care. J Allergy Clin Immunol 114: 155-212, 2004.

5. Soler ZM, Sauer D, Mace J and Smith TL: Impact of mucosal eosinophilia and nasal polyposis on quality-of life outcomes after sinus surgery. Otolaryngol Head Neck Surg 142: 64-71, 2010.

6. Vlaminck S, Vauterin T, Hellings PW, Jorissen M, Acke F, Van Cauwenberge P, Bachert $\mathrm{C}$ and Gevaert P: The importance of local eosinophilia in the surgical outcome of chronic rhinosinusitis: A 3-year prospective observational study. Am J Rhinol Allergy 28: 260-264, 2014.

7. Wang X, Zhang, Bo M, Holtappels G, Zheng M, Lou H, Wang H, Zhang L and Bachert C: Diversity of TH cytokine profiles in patients with chronic rhinosinusitis: A multicenter study in Europe, Asia, and Oceania. J Allergy Clin Immunol 138: 1344-1353, 2016.

8. Lou H, Meng Y, Piao Y, Wang C, Zhang L and Bachert C: Predictive significance of tissue eosinophilia for nasal polyp recurrence in the Chinese population. Am J Rhinol Allergy 29: 350-356, 2015.

9. Nakayama T, Yoshikawa M, Asaka D, Okushi T, Matsuwaki Y, Otori N, Hama T and Moriyama H: Mucosal eosinophilia and recurrence of nasal polyps-new classification of chronic rhinosinusitis. Rhinology 49: 392-396, 2011.

10. Cho SW, Kim DW, Kim JW, Lee CH and Rhee CS: Classification of chronic rhinosinusitis according to a nasal polyp and tissue eosinophilia: Limitation of current classification system for Asian population. Asia Pac Allergy 7: 121-130, 2017.

11. Zhang N, Van Zele T, Perez-Novo C, Van Bruaene N, Holtappels G, DeRuyck N, Van Cauwenberge P and Bachert C: Different types of T-effector cells orchestrate mucosal inflammation in chronic sinus disease. J Allergy Clin Immunol 122: 961-968, 2008.

12. Katotomichelakis M, Tantilipikorn P, Holtappels G, De Ruyck N, Feng L, Van Zel T, Muangsomboon S, Jareonchasri P, Bunnag C, Danielides V, et al: Inflammatory patterns in upper airway disease in the same geographical area may change over time. Am J Rhinol Allergy 27: 354-360, 2013.

13. Sakuma Y, Ishitoya J, Komatsu M, Shiono O, Hirama M, Yamashita Y, Kaneko T, Morita S and Tsukuda M: New clinical diagnostic criteria for eosinophilic chronic rhinosinusitis. Auris Nasus Larynx 38: 583-588, 2011.

14. Cao PP, Li HB, Wang BF, Wang SB, You XJ, Cui YH, Wang DY, Desrosiers $\mathrm{M}$ and Liu Z: Distinct immunopathologic characteristics of various types of chronic rhinosinusitis in adult Chinese. J Allergy Clin Immunol 124: 478-484, 2009.

15. Wang ZC, Yao Y, Wang N, Liu JX, Ma J, Chen CL, Deng YK, Wang MC, Liu Y, Zhang XH and Liu Z: Deficiency in interleukin-10 production by $\mathrm{M} 2$ macrophages in eosinophilic chronic rhinosinusitis with nasal polyps. Int Forum Allergy Rhinol 8: 1323-1333, 2018

16. Perić A, Baletić N, Sotirović J and Špadijer-Mirković C: Macrophage inflammatory protein-1 production and eosinophil infiltration in chronic rhinosinusitis with nasal polyps. Ann Otol Rhinol Laryngol 124: 266-272, 2015. 
17. Bachert C, Wagenmann M, Hauser U and Rudack C: IL-5 synthesis is upregulated in human nasal polyp tissue. J Allergy Clin Immunol 99: 837-842, 1997.

18. Meyer JE, Bartels J, Görögh T, Sticherling M, Rudack C, Ross DA and Maune $S$ : The role of RANTES in nasal polyposis. Am J Rhinol 19: 15-20, 2005.

19. Okada N, Nakayama T, Asaka D, Inoue N, Tsurumoto T, Takaishi S, Otori N, Kojima H, Matsuda A, Oboki K, et al: Distinct gene expression profiles and regulation networks of nasal polyps in eosinophilic and non-eosinophilic chronic rhinosinusitis. Int Forum Allergy Rhinol 8: 592-604, 2018.

20. Wang W, Gao Z, Wang H, Li T, He W, Lv W and Zhang J: Transcriptome analysis reveals distinct gene expression profiles in eosinophilic and noneosinophilic chronic rhinosinusitis with nasal polyps. Sci Rep 6: 26604, 2016.

21. Newman AM, Liu CL, Green MR, Gentles AJ, Feng W, Xu Y, Hoang CD, Diehn $M$ and Alizadeh AA: Robust enumeration of cell subsets from tissue expression profiles. Nat Methods 12: 453-457, 2015

22. Wei TY and Simko V: R package 'corrplot': Visualization of a Correlation Matrix (version 0.84). https://github. com/taiyun/corrplot.

23. Langfelder $P$ and Horvath S: WGCNA: An R package for weighted correlation network analysis. BMC Bioinformatics 9: $559,2008$.

24. Zhang B and Horvath S: A general framework for weighted gene co-expression network analysis. Stat Appl Genet Mol Biol 4: 17, 2005.

25. Langfelder P, Zhang B and Horvath S: Defining clusters from a hierarchical cluster tree: The dynamic tree cut package for $\mathrm{R}$ Bioinformatics 24: 719-720, 2008.

26. Livak KJ and Schmittgen TD: Analysis of relative gene expression data using real-time quantitative PCR and the 2(-Delta Delta C(T)) method. Methods 25: 402-408, 2001.

27. Yu G, Wang LG, Han Y and He QY: ClusterProfiler: An R package for comparing biological themes among gene clusters. OMICS 16: 284-287, 2012.

28. MacLeod MK, Clambey ET, Kappler JW and Marrack P: CD4 memory T cells: What are they and what can they do? Semin Immunol 21: 53-61, 2009

29. Turner DL, Bickham KL, Thome JJ, Kim CY, D'Ovidio F, Wherry EJ and Farber DL: Lung niches for the generation and maintenance of tissue-resident memory T cells. Mucosal Immunol 7: 501-510, 2014.

30. Teijaro JR, Turner D, Pham Q, Wherry EJ, Lefrançois L and Farber DL: Cutting edge: Tissue-Retentive lung memory CD4 $\mathrm{T}$ cells mediate optimal protection to respiratory virus infection J Immunol 187: 5510-5514, 2011.

31. Nakanishi Y, Lu B, Gerard C and Iwasaki A: CD8(+) T lymphocyte mobilization to virus-infected tissue requires CD4(+) T-cell help. Nature 462: 510-513, 2009.

32. Shenoy AT, Wasserman GA, Arafa EI, Wooten AK, Smith NM, Martin IM, Jones MR, Quinton LJ and Mizgerd JP: Lung $\mathrm{CD} 4+$ resident memory $\mathrm{T}$ cells remodel epithelial responses to accelerate neutrophil recruitment during pneumonia. Mucosal Immunol 13: 334-343, 2019.

33. Wu D, Wang J and Zhang M: Altered Th17/Treg ratio in nasal polyps with distinct cytokine profile association with patterns of inflammation and mucosal remodeling. Medicine (Baltimore) 95: e2998, 2016

34. Bonneville M, O'Brien RL and Born WK: Gammadelta T cell effector functions: A blend of innate programming and acquired plasticity. Nat Rev Immunol 10: 467-478, 2010.

35. Shapouri-Moghaddam A, Mohammadian S, Vazini H, Taghadosi M, Esmaeili SA, Mardani F, Seifi B, Mohammadi A, Afshari JT and Sahebkar A: Macrophage plasticity, polarization, and function in health and disease. J Cell Physiol 233: 6425-6440, 2018.

36. Hua X, Naselsky WC, Jania CM, Chason KD, Huang JJ, Doerschuk CM, Graham SM, Senior BA and Tilley SL: Mas cell deficiency limits the development of chronic rhinosinusitis in mice. Ann Otol Rhinol Laryngol 125: 290-296, 2016.
37. Gröger M, Bernt A, Wolf M, Mack B, Pfrogner E, Becker S and Kramer MF: Eosinophils and mast cells: A comparison of nasal mucosa histology and cytology to markers in nasal discharge in patients with chronic sino-nasal diseases. Eur Arch Otorhinolaryngol 270: 2667-2676, 2013.

38. Kim JH, Choi GE, Lee BJ, Kwon SW, Lee SH, Kim HS and Jang YJ: Natural killer cells regulate eosinophilic inflammation in chronic rhinosinusitis. Sci Rep 6: 27615, 2016.

39. Gelardi M, Piccininni K, Quaranta N, Quaranta V, Silvestri M and Ciprandi G: Olfactory dysfunction in patients with chronic rhinosinusitis with nasal polyps is associated with clinical-cytological grading severity. Acta Otorhinolaryngol Ital 39: 329-335, 2019.

40. Lavin J, Min JY, Lidder AK, Huang JH, Kato A, Lam K, Meen E, Chmiel JS, Norton J, Suh L, et al: Superior turbinate eosinophilia correlates with olfactory deficit in chronic rhinosinusitis patients. Laryngoscope 127: 2210-2218, 2017

41. Shah SA, Ishinaga $\mathrm{H}$ and Takeuchi K: Pathogenesis of eosinophilic chronic rhinosinusitis. J Inflamm (Lond) 13: 11, 2016.

42. Clutterbuck E, Shields JG, Gordon J, Smith SH, Boyd A Callard RE, Campbell HD, Young IG and Sanderson CJ: Recombinant human interleukin 5 is an eosinophil differentiation factor but has no activity in standard human B cell growth factor assays. Eur J Immunol 17: 1743-1750, 1987.

43. Clutterbuck EJ, Hirst EM and Sanderson CJ: Human interleukin-5 (IL-5) regulates the production of eosinophils in human bone marrow cultures: Comparison and interaction with IL-1, IL-3, IL-6, and GMCSF. Blood 73: 1504-1512, 1989.

44. Clutterbuck EJ and Sanderson CJ: Regulation of human eosinophil precursor production by cytokines: A comparison of recombinant human interleukin-1 (rhIL-1), rhIL-3, rhIL-5, rhIL-6, and rh granulocyte-macrophage colony-stimulating factor. Blood 75: 1774-1779, 1990.

45. Sauter A, Stern-Straeter J, Chang RC, Hörmann K and Naim R: Influence of interleukin-13 on beta-catenin levels in eosinophilic chronic rhinosinusitis cell culture. Int J Mol Med 21: 447-452, 2008.

46. Novak N and Donald YM: Role of barrier dysfunction and immune response in atopic dermatitis. In: Pediatric Allergy: Principles and Practice. 3rd edition. Elsevier, pp438-447, 2016.

47. Alobid I and Mullol J: Role of medical therapy in the management of nasal polyps. Curr Allergy Asthma Rep 12: 144-153, 2012.

48. Takeda K, Takeno S, Hirakawa K and Ishino T: Expression and distribution of glucocorticoid receptor isoforms in eosinophilic chronic rhinosinusitis. Auris Nasus Larynx 37: 700-707, 2010.

49. Pujols L, Mullol J, Benítez P, Torrego A, Xaubet A, de Haro J and Picado C: Expression of the glucocorticoid receptor alpha and beta isoforms in human nasal mucosa and polyp epithelial cells. Respir Med 97: 90-96, 2003.

50. Wang H, Bai J, Ding M, Liu W, Xu R, Zhang J, Shi J and $\mathrm{Li} \mathrm{H}$ : Interleukin-17A contributes to the expression of serum amyloid A in chronic rhinosinusitis with nasal polyps. Eur Arch Otorhinolaryngol 270: 1867-1872, 2013.

51. He R, Sang H and Ye RD: Serum amyloid A induces IL-8 secretion through a $\mathrm{G}$ protein-coupled receptor, FPRL1/LXA4R. Blood 101: 1572-1581, 2003.

52. Choi GE, Yoon SY, Kim JY, Kang DY, Jang YJ and Kim HS: Autophagy deficiency in myeloid cells exacerbates eosinophilic inflammation in chronic rhinosinusitis. J Allergy Clin Immunol 141: 938-950, 2018.

53. Wang BF, Cao PP, Wang ZC, Li ZY, Wang ZZ, Ma J, Liao B, Deng YK, Long XB, Xu K, et al: Interferon- $\gamma$-induced insufficient autophagy contributes to p62-dependent apoptosis of epithelial cells in chronic rhinosinusitis with nasal polyps. Allergy 72: 1384-1397, 2017.

This work is licensed under a Creative Commons Attribution-NonCommercial-NoDerivatives 4.0 International (CC BY-NC-ND 4.0) License. 